\title{
DISTRIBUTED SIMULATION FOR TASK-DRIVEN FLEXIBLE MANUFACTURING SYSTEMS
}

\author{
PESCHL, M[ichael]; ROENING, J[uha] \& LINK, N[orbert]
}

\begin{abstract}
In this paper a novel approach for distributed simulation and emulation of manufacturing tasks which is embedded in the new paradigm of task-driven manufacturing is proposed. The core of the approach is the Production Simulation System which is an offline software tool that issues simulation jobs and performs multi-dimensional cost estimation and optimization. The simulation itself is done by intelligent production units called Manufactrons or by standard simulation tools. The main benefit of the approach is that the process configuration on shop floor is taken into account during simulation. The new approach has successfully been tested in two application scenarios.
\end{abstract}

Keywords: simulation, emulation, manufacturing, task-driven, eBOP, production simulation system

\section{INTRODUCTION}

Nowadays manufacturing systems are faced by different challenges. One of the most important requirements is the implementation of flexible manufacturing systems (FMS). Such systems target on the adaptation of the manufacturing process, e.g. on different production volumes and product variants in order to satisfy the upcoming needs for customer-tailored products and for decreasing the lot sizes down towards lot size one (see also [1]).

In order to reach this goal while also keeping the costs in an acceptable frame, several pre-conditions and boundary conditions have to be taken into account. This paper concentrates on a.) the need for fast ramp-up of single production equipment and $b$.) the need for optimal production configuration and optimization. The first need mainly targets on the capabilities of the production equipment in terms of self-configurability and selfdescription. Machines and devices are required which are capable to adapt themselves rapidly to different production conditions. The second need for optimal production configuration and optimization concentrates on the orchestration of production equipment. In addition to the more traditional optimization targets such as throughput, also other optimization goals, e.g. energy consumption or $\mathrm{CO}_{2}$ emission become more and more important [2].

Obviously, both needs are highly interrelated: In order to obtain optimal production configuration on cell or line level, the setup of the single machines and devices must fit into the (global) optimization concepts and targets. Otherwise only local optimization is possible.

\section{STATE OF THE ART}

In order to speed up the ramp-up of production many approaches do exist. One of the most popular concepts is the Digital Factory. The core of this concept is the integration of digital models, methods and visualization through a comprehensive data and management [3]. Digital models, planning and simulation build the Virtual Factory which is integrated into the real factory by data coupling of virtual and real factory. The models within the Virtual Factory are concurrently proven and thus, the ramp-up time of production can significantly be reduced.

While for product design many tools are already existing, lacks of approaches for the integration of tools, planning levels and optimization on a multi criteria level do exist. Furthermore, significant efforts and research activities are required for operative production planning and control down to the factory floor [4].

In [5] a standard framework and in [6] a reference model for simulating and modeling manufacturing systems is proposed. However, interfacing ERP and SCADA for real-time updates for simulation parameters and a formalization for a standard representation and functioning of manufacturing production processes is not implemented yet.

Most of the existing FMS and the related simulation models concentrate on the traditional target dimensions of optimization. In [2] an approach for taking also environmental targets into account is described. However, the calculation of the impact on the environment is done in Excel worksheets only.

The literature survey clearly indicates that a lot of approaches for (distributed) simulation exist. However, they either concentrate on the implementation of dedicated process simulation in specific tools or on the specification and/or implementation of frameworks which lack of the integration of real production data. Even if approaches for standardized interfaces do exist (e.g. STEP), many tools only implement proprietary interfaces.Summarizing the said before, a holistic approach for finding

1. an optimal production configuration for fulfilling the desired production goals,

2. a setup for a global, factory-wide optimization under various optimization targets,

3. an optimal configuration for the single machines and devices which also fits into the global optimization goals

is required. Technically spoken, a tool is required whose output is the arrangement and setup of production machines and device for factory-wide optimal production. The tool will be used "offline" tool in the design phase of the production process. However, in order to obtain maximum benefit, the output of the tool 
shall be used directly for production (equipment) configuration without further processing.

\section{TASK-DRIVEN PRODUCTION}

The task-driven production is a new paradigm in manufacturing. In contrast to the traditional recipe-based techniques, the machines and devices on shop floor only receive a description of the task they shall perform. By this method, the machines are executing the task without further information from outside. Machines and devices with such properties are called Manufactrons [7]. The jobs are issued by the Production Configuration System on ERP level and are communicated by the Workflow Managers on MES level (Fig. 1).

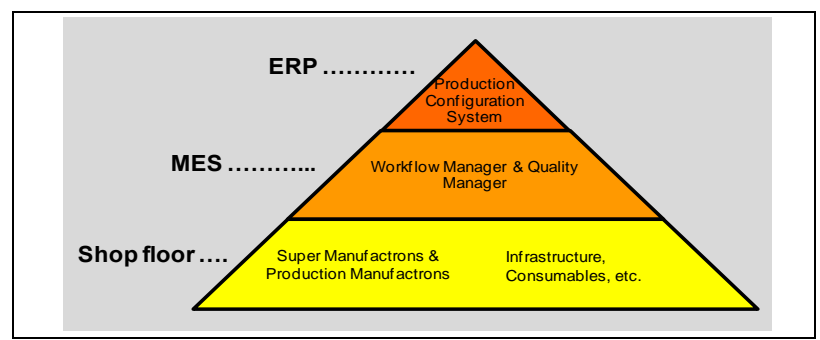

Fig. 1 Hierarchical organization

During the production phase, Manufactrons receive task descriptions in order to execute a production job (e.g. perform a transport or handling task, do a joining or assembly operation). The challenge in the production layout design and production ramp-up phase is to find the optimal layout configuration and task descriptions for the Manufactrons on shop floor with respect to the production goals and boundary conditions.

\section{DISTRIBUTED EMULATION AND SIMULATION}

\subsection{Concept}

In order to overcome the limitations of current existing approaches, a novel concept of distributed simulation and emulation is proposed. The concept is embedded into the paradigm of task-driven manufacturing. The approach is characterized by a holistic concept for integrating simulation on the highest level of factory planning down to dedicated process simulations. By this, an optimal production configuration with respect to the optimization targets is realized. During production, also improvements for production scheduling as well as for bottleneck identification are possible.

The basic idea of the novel approach is to upgrade machines and devices by Manufactrons that are able to simulate and emulate their own behavior with respect to the desired target. For doing so, the Manufactrons receive simulation jobs from a superordinate job scheduler. The simulation job consists of information on the simulation target and the respective boundary conditions. After simulation, the Manufactrons submit their simulation results to the simulation job scheduler. By gathering and assessing the simulation results of each single Manufactrons, a global simulation and optimization of a (virtual) production scenario is reached. The final goal of each simulation job is the compilation of a template for the electronic Bill Of Processes (eBOP) for the production of one product variant. In the definition of the
Manufactronic Factory the eBOP for one product variant is called a method.

\subsection{Production Simulation System}

The core of the novel approach is the Production Simulation System. This software tool is a part of the socalled Production Configuration System which resides on the top level of the manufacturing pyramid (Fig. 1). The Production Simulation System is responsible for issuing simulation jobs to the Manufactrons underneath on shop floor level and for receiving and assessing their simulation results.

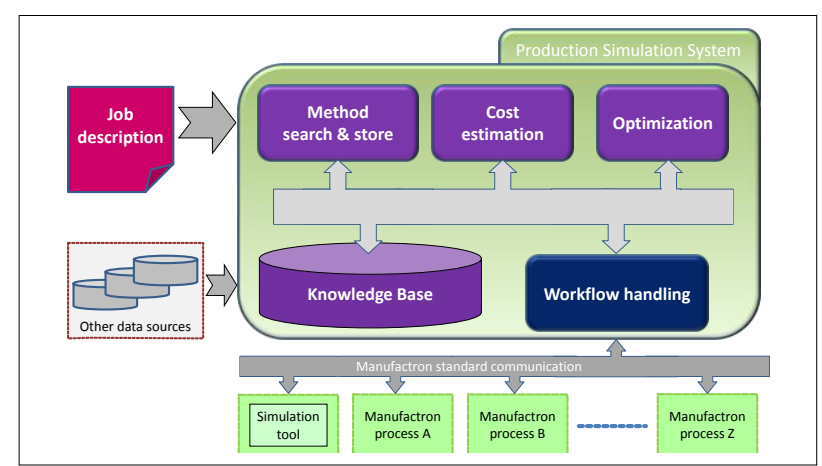

Fig. 2 Production Simulation System

The Production Simulation System consists of several building blocks (Fig. 2) for performing its work:

The knowledge base consists of already available methods for production setups. Those methods are the result of simulation jobs performed in the past. The methods might be used as a starting point for new simulations in order to decrease the simulation time.

The method search\&store block is responsible for the method handling of the Knowledge Base. It searches proper methods within the Knowledge Base on the basis of a job description which is specified by a user. If new simulation methods are available, the method search\&store block is also responsible for inserting the new method into the Knowledge Base.

The cost estimator gathers all simulation results from the Manufactrons. This contains relevant data such as task execution time, resource consumption and process quality. The cost estimator also assesses those data by means of a user-defined cost function giving weights to all performance numbers, where also the space and building infrastructure needed to maintain a process can be included. The latter as well as the weighting functions can be entered via a cost definition system.

Based on the cost function, the optimum configuration of the setup for the targeted variety of products will be found by the optimization system. The workflow handling_is responsible for controlling the workflow during simulation. It issues simulation jobs to the respective Manufactrons and receives their simulation results. For Manufactron addressing it has an interface to a data dictionary.

\subsection{Process emulation and simulation on machine level}

As described before, Manufactrons are equipped with simulation and emulation capabilities. This enables the Manufactrons not only to execute tasks, but also to answer requests for simulation and emulation. Similar to 
the Production Simulation Systems, Manufactrons also consist of a knowledge base which stores methods. Those methods describe the proper setup of the respective device with respect to a task description.

In this context emulation is defined as the (virtual) execution of an already existing method of the Manufactron's knowledge base with respect to the respective emulation target. In contrast to that, simulation means the generation of new methods.

When the Manufactron receives a simulation task, it queries its knowledge base for proper methods. The best fitting method is used to perform an emulation. If the result of the emulation corresponds with the required target (e.g. time consumption, process quality, etc.), the emulation result is submitted to the Production Simulation System for further processing. If no proper method is available, a new simulation job is performed by the Manufactron. The outcome of the simulation (the process method) is inserted in the Manufacton's knowledge base and the requested KPI's are submitted to the Production Simulation System. The detailed sequence of the simulation steps is illustrated in the next figure.

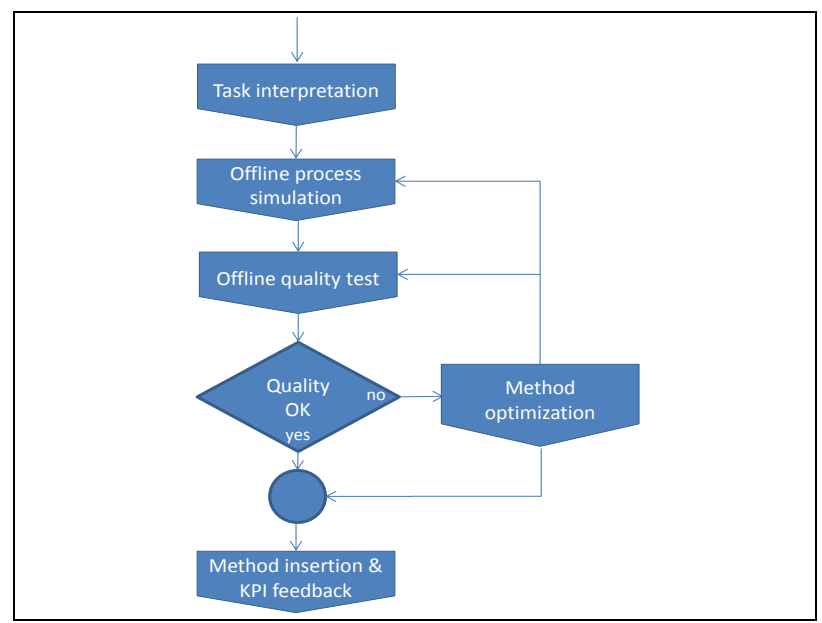

Fig. 3 Sequence of simulation steps

As the Knowledge Bases of the Manufactrons are connected to each other, a new method which is generated by simulation by one Manufactron is immediately available for all other Manufactrons of the same type. By this behavior, a learning network of Manufactrons is created in which always the best and newest methods for production execution are present for each entity connected.

\subsection{Embedding of simulation tools}

The section above describes the capabilities of the Manufactrons for simulation. However, a lot of (commercial) off-the-shelf and dedicated software solutions do exist for simulation. Various software packages for production planning, virtual commissioning as well as dedicated process simulation do exist on the market. Thus, it does not make sense to re-implement those capabilities in the Manufactrons. For that reason, simulation and emulation capabilities might not be an intrinsic element of the Manufactrons.

The novel approach for distributed simulation and emulation provides two ways for embedding simulation tools into the system. Prerequisite for both ways is that the software tools are equipped with a programming interface allowing to trigger simulation jobs and to feedback the simulation results.

The first approach is to interface the simulation tool from the respective Manufactron. When a Manufactron receives a request for simulation, it forwards the request to the simulation tool. After the tool executed the simulation, the Manufactron obtains the result and submits it to the Production Simulation System. This approach might be useful for very dedicated simulation tools (e.g. dedicated process simulation such as parameter finding of a welding or riveting process).

The second approach is to wrap the simulation tool by a Manufactronic shell. In this case, the simulation tool becomes to a "Simulation Manufactron" which receives a task description, performs the task (simulation/ emulation) and issues the result. The main advantage of this solution is the standard communication mechanism which is provided by the Manufactronic communication scheme. Simulation Manufactrons can be integrated into various simulation scenarios and setups without any reprogramming and other adaptation. This approach is most useful for interfacing complex tools which are able to simulate e.g. entire factory setups.

\subsection{Example sequence of simulation}

In the previous sections the building blocks of the approach have been illustrated. Following, a typical sequence is described for illustrating their interaction.

1. The user defines a job description (the product to be produced, machines available, etc.).

2. The Production Simulation System queries its knowledge base in order to find already existing methods for fulfilling the job description. Those methods consist of the setup of the production (e.g. number and arrangement of machines, production sequence) as well as of the setup of the dedicated machines and devices (e.g. parameters, control values). By calculating a "similarity value", the user obtains a feedback on the "quality" of the found method with respect to the inserted job description. If the quality of the found method is sufficient enough, no further action is required. The method (the eBOP) can be used for production without further adaptation. However, in the usual cases, improvements in the eBOP will be required. In these cases, the best fitting method found in the knowledge base is used as a basis for a new simulation job.

3. By issuing dedicated simulation jobs the Production Simulation System "asks" all Manufactrons for their offered optimum methods with respect to the necessary and implicitly given tasks.

4. The Manufactrons interpret the task descriptions and perform an emulation job or, if required, also a simulation job (see section 4.3) and issue the results to the Production Simulation System.

5. The simulation results are assessed with respect to their profiles and requirements by the cost estimator which represents the target function (for each product type) to be achieved by the production process.

6. Based on the cost function, the optimum configuration of Manufactrons for the targeted variety of products will be found by the cost-function optimisation system. The sequence with the lowest cost is then the eBOP of the specific product type and is inserted into the Knowledge Base. 


\section{APPLICATIONS}

The novel approach for distributed emulation and simulation has successfully been tested in two application scenarios.In the first scenario a production line of 12 virtual Manufactrons (representing 12 stations) for the assembling of photovoltaic wafers has been built. Within eleven test cases, the various simulation jobs have been issued by the Production Simulation System to the virtual Manufactrons. The major goal of the scenario was to test the systems in terms of its capability for i.) job retrieval, similar job querying and eBOP generation out of the Knowledge base, ii.) issuing simulation and emulation jobs to the virtual Manufactrons and to the external simulation tool for line simulation and iii.) assessing and optimizing the simulation results by the internal blocks cost estimator and optimizer.

The second scenario focused on the embedding of dedicated simulation tools for robot path generation and welding parameter finding. The major goal was to discover the optimal setup of configuration of a robot and a welding control unit in an automotive production cell.

\section{SYSTEM BENEFITS}

The results of the system tests within the application scenarios described above were very promising. It could be demonstrated, that the system fits perfectly in the holistic approach of Manufactronic manufacturing. The system provides the following major advantages:

- The integration of production flow simulation on MES level and process simulation on shop floor level provides a multi-dimensional, factory-wide optimization of the production process.

- The automatic generation of eBOP template during the simulation decreases the production setup design time and ramp-up time significantly.

- Expert knowledge is conserved in the knowledge base of the Production Simulation System and on the level of process simulation within the production Manufactrons. The newest methods are always available and accessible by all other Manufactrons.

- Commercial simulation tools can be embedded easily by the implementation of standard interfaces and communication mechanisms.

- Suppliers can provide virtual Manufactrons which can be used for simulation purposes in advance of the real production (e.g. during the design phase). After that, the virtual Manufactrons can be replaced by real Manufactrons without any further configuration and adaptation efforts. This enables for real plug \& produce capabilities.

- The Production Simulation System provides a userfriendly HMI for performing distributed simulation and emulation jobs. It provides an interface to access its knowledge base and eBOP templates.

\section{CONCLUSION}

In this paper a novel approach for distributed simulation and emulation which fits smoothly in the new paradigm of task-driven manufacturing is presented. The core of the approach is the Production Simulation System which is capable to issue emulation and simulation jobs to the (virtual) production Manufactrons underneath. It allows performing multi-dimensional optimization tasks based on the simulation results of the Manufactrons on shop floor. The resulting eBOP template and the related expert knowledge can be conserved and made accessible to other users.

Further research will concentrate on the reduction of complexity jobs by the introduction of hierarchical simulation and emulation based on the approach of the Super Manufactrons. In addition to that improvements of the operability of the Production Simulation System and the parallel execution of simulation jobs will be in focus.

\section{ACKNOWLEDGEMENTS}

This study was carried out with financial support from the European Commission under the Sixth Framework Programme for Research and Technological Development in Integrated Project XPRESS (Flexible Production Experts for Reconfigurable Assembly Technology, IP 026674-2); Project homepage: xpressproject.eu. Special thanks go to Critical Manufacturing, Porto, Portugal for their investigation in database configuration and querying, for programming the Production Simulation System and for setting up the first test scenario. Thanks also to KUKA Systems, Augsburg, Germany for providing the test environment for the second scenario. Thanks also to Fraunhofer IPA, Stuttgart, Germany and to the University of Porto, Portugal for their contributions.

\section{REFERENCES}

[1] Nylund, H.; Salminen, K. \& Andersson, P. (2008). Modelling and Simulation in the Context of Distributed Manufacturing Systems, Annals of DAAAM 2008, pp.927-928, 22-25th October 2008, ISSN 1726-9679, Trnava, Slovakia

[2] Heilala, J.; Vatanen, S.; Tonteri, H.; Montonen, J.; Lind, S.; Johansson, B.; Stahre, J. (2008): Simulation-based sustainable manufacturing system design. Proceedings of the 2008 Winter Simulation Conference, Miami, Florida, ISBN: 978-1-4244-27086, S. J. Mason, R. R. Hill, L. Mönch, O. Rose, T. Jefferson, J. W. Fowler S. (Ed.) pp. 1922-1930, WDC, Piscataway, NJ

[3] Westkämper, E.; Gottwald, B.; Fisser, F. (2005). Migration of the digital and virtual factory to reality. CIRP Journal of Manufacturing Systems, Vol 34, No. 5, pp. 391-396, ISSN 02786125

[4] Kuehn, W. (2006). Digital factory - Integration of simulation enhancing the product and production process towards operative control and optimization. International Journal of Simulation Vol 7 No.7, pp. 27-39

[5] Battista, C, Giordano, F., Iannone, R., Schiraldi, M (2010). A proposal for a standard framework for simulating and modeling manufacturing systems. Proceedings of the Conference on 'Sustainable Development: Industrial Practice, Education \& Research' 2010, Monopoli, BA (Italy), September 2010, ISSN 978-88-904625-1-1

[6] Battista, C.; Dello Stritto, G.; Giordano, F.; Iannone, R. \& Schiraldi, M. (2011) Manufacturing Systems Modelling and Simulation Software Design: a Reference Model, Annals of $D A A A M$ for 2011 \& Proceedings of the $22^{\text {nd }}$ International DAAAM Symposium, ISBN 978-3-901509-83-4, ISSN 17269679, Katalinic, B. (Ed) , pp 1107-1108 DAAAM International, Vienna, Austria

[7] Peschl, M. \& Hoffmeister, M. (2011). A Task-Driven Flexible Manufacturing System for Major Industrial Applications, Annals of DAAAM for 2011 \& Proceedings of the 22nd International DAAAM Symposium, 23-26th November 2011, Vienna, Austria, Volume 22, No. 1, ISSN 1726-9679, ISBN 978-3-901509-83-4, Katalinic, B. (Ed.), pp. 1463-1464, DAAAM International Vienna, Austria 